\title{
MOVABLE SPLEEN.
}

\author{
By T. G. MOORHEAD, M.D. UNIV. IUB., F.R.C.P.I. : \\ Physician to the Royal City of Dublin Hospital.
}

[Read in the Section of Medicine. March 1. 1907.]

Excessive mobility of the spleen and dislocation of the spleen from its normal position are conditions of considerable rarity, judging from the records scattered through the literature, but they are still sufficiently often met with to render a remembrance of their possibility most important in abdominal diagnosis. In common with the other solid viscera which are in contact with the under surface of the diaphragm, the spleen moves up and down with the respiratory contractions of that muscle, and it is consequently necessary to limit the term movable, floating or wandering spleen to cases in which the organ has actually slipped or is capable of slipping from its usual bed into some other part of the abdomen. The additional term dislocation of the spleen is occasionally used. and is confined to cases in which the spleen, after leaving its normal site, becomes attached by adhesions in some other spot and again loses its mobility.

The ætiology of the condition has happily not given rise to so many theories nor to so much discussion as that which has centred round the more commonly met with movable kidney, and this is probably due to the fact that, as a rule, definite pathological changes can be detected, in most cases of movable spleen, when such come under observation, either at operation or at autopsy. The most important of these changes, from the ætiological point of view, are as follows :-

(1) Enlargement.-All writers are agreed in stating that a 
movable spleen is almost always bigger than normal, but that this is not an invariable condition is proved by the occasional record of cases in which no enlargement is found. Such cases have been put on record by Freudenberg' ${ }^{\mathbf{l}}$, Martin, and others. In considering enlargement, also, it is necessary to recollect that the dislocation itself, by leading to pressure upon and twisting of the splenic vessels, may cause great passive engorgement, and that thus the large size may be a secondary and not a primary phenomenon. Still the fact remains that in most cases a cause for enlargement has existed before displacement became noticeable.

(2) Relaxation of the splenic Ligaments.-This condition is, of course, necessarily present in order that displacement at all may occur, and will naturally be favoured by any cause, such as enlargement of the organ, which puts an unusual strain upon them. It has also been suggested that relaxation may result as a developmental defect, while its occurrence in cases of general splanchnoptosis is to be expected. In this connection it may also be mentioned that a movable left kidney is liable to be followed by general loosening of the peritoneum in that neighbourhood, and may thereby come to exert an important causative influence. This last statement is illustrated by the case that I am bringing before you to-night, and also by a case reported by Grieffenhagen" in which, some time after a nephropexy of the left kidney had been performed. the condition apparently returned, but, on operation, turned out to be a floating spleen. Is a factor in complicating the diagnosis, previous linowledge of the existence of a movable kidney is most important.

(3) Trauma.-This probably acts by causing a rupture of the splenic ligaments, and at the same time a sudden dislocation of the spleen. Its importance is borne out by many reported instances, but the most striking of these that $I$ have come across is a case reported by Ssawiljew ${ }^{3}$ in a perfectly 
healthy boy, aged four years, in whom a greatly displaced spleen was found immediately subsequent to a severe fall.

Before leaving the subject of ætiology it may be as well to refer to the fact that the condition is much more common in women, and is particularly found in those who have a relaxed abdominal wall.

The displaced viscus may be found in almost any part of the abdomen, and has even located itself in such an unlikely spot as the right iliac fossa, but the most common places for it to occupy are the cavity of the true pelvis, and the left iliac fossa. Within the pelvis it may give rise to numerous disturbances, and has simulated and frequently been mistaken for tumours and cysts of the uterus and its appendages. In the left iliac fossa it is most likely to be mistaken for a renal tumour, but here also it may resemble an ovarian cyst, or some growth of the mesentery or omentum. Occasionally even a sense of fluctuation has been detected in the lump, as in a case recorded by Croly $y^{4}$ in 1896, and in such a case the diagnostic difficulties would be augmented. At first sight it may seem curious that difficulties in diagnosis should exist at all, as the shape of the spleen is so characteristic, but when it is remembered that displacement is always accompanied by axial rotation, and also by engorgement, which latter tends to alter the shape, this difficulty is easily explicable. Again, the normal shape of the spleen is regarded as being largely produced by the pressure and counter pressure of adjacent viscera, and therefore $I$ think we are justified in concluding that some change of shape will result from an alteration of the manner and direction in which those forces are applied. The axial rotation is of such a nature as to bring the diaphragmatic surface downwards and forwards, and consequently to cause the characteristically shaped borders and edges to be turned away from the examining hand. What relation the spleen maintains as a rule to the colon I have 
been unable to determine by a search through the literature, but my own case shows that it may, at any rate, at times slip down posterior to the descending colon. The rotation that occurs may sometimes result in an actual twisting of the pedicle, and when this has taken place thrombosis of the splenic veins or artery, atrophy of the spleen, complete necrosis, or even complete separation of the spleen from all attachments may occur. More usually, however, none of these accidents take place, and the symptoms are entirely referable to pressure upon neighbouring viscera. Such pressure may prove serious, and even give rise to intestinal obstruction.

The case which I wish to bring before you to-night, and which has given rise to the foregoing remarks, was one of a movable spleen situated in the left iliac fossa, and in which the diagnosis was only made at operation. It, in fact, presented us with a typical example of " the astonishment which follows the discovery of these movable spleens in the operating theatres," and to which Bland Sutton ${ }^{5}$ graphically refers in his interesting paper on this subject. The notes of it are as follow :-

L. G., a girl, unmarried, aged twenty-nine, first came under my care about two and a half rears ago suffering from serious symptoms of cardiac failure. There was a history of numerous attacks of rheumatic fever and of long-standing cardiac trouble. Some years previously she had had a severe fall from a ladder, which had laid her up for some weeks, but which did not appear to have led to any permanent ill effects. Two months before her admission to the (ity of Dublin Hospital her left knee had been excised at another Dublin hospital for what she stated to be, and for what presumably was, tubercular clisease.

Examination at the time revealed the presence of double mitral disease and of adherent pericardium, and in the abdomen some slight liver enlargement was noted. In addition, the left kidney was freely movable, and its entire shape and size could be made out with little difficulty, although the abdominal wall was 
quite well developed. On mentioning. in the patient's presence, that her kidney was movable she informed me that a doctor who had examined her several years previously had told her the same. The spleen was not palpable, but the area of dulness corresponding to it was rather more extensive than one usually finds. The urine at this time contained albumen, but no pus. Nothing else noteworthy was present; the patient rapilly improved, and left hospital after six weeks.

During the next couple of years she was re-admitted on three different occasions. On the first two of these her symptoms did not differ from those above referred to, but on the third oc'asion she complained somewhat of pain in the left side of the abdomen. and on palpating here a distinct lump conld be felt, which moved freely up and down, and was exquisitely tender on pressure. The tumour felt about twice the size of a normal kidner, but it rapidly decreased in volume, and nothing at all could be felt at the time the patient left hospital, two months subseçuent to her admission.

I did not see her again for six months, when she was again admitted suffering from cardiac asthma and complaining very much of her left side. Examination now revealed the presence of a tumour in the left lumbar and left iliac regions, and of a much greater size than it had been on the previous occasion. The lower margin of it conld be distinctly felt to be rounded, and an obscure sense of fluctuation was obtained over it. The upper margin disappeared under the costal arch and could not be felt. On palpating the growth it was found to move up and down slightly, while percussion revealed the presence of intestine anterior to it, and yielded a dull note at the side, from about the line of the anterior axillary fold back to the spine. It was still intensely tender to touch, and severe paroxysms of pain, lasting often for over an hour, were experienced in the region once or twice daily. The urine was still albuminous, but free from pus, and the only other symptom present was great frequency of micturition, but without bladder pain. The temperature was normal, and there was no leucocytosis.

The diagnosis that $I$ arrived at was that of hydronephrosis secondary to movable kidney, and, as I thought that surgical intervention might become necessary, I asked my 
rolleague, Mr. Stoney, to see the case with me. He concurred in the diagnosis, and, at my request, made a cystoscopic examination. This was necessarily unsatisfactory, as it was thought inadvisable to give an anæsthetic, and the patient's condition made her extremely sensitive, but as far as it went it was in favour of the diagnosis otherwise arrived at, as no cystitis was found to account for the frequency of micturition, and the quantity of urine obtained from the left kidney by the segregation method was less than from the right. We, however, decided that, having regard to the condition of the heart, it was better not to operate unless signs of suppuration within the sac occurred. This determination seemed justified by the subsequent course of the case, as within three days the tumour noticeably diminished in size, and finally almost completely disappeared. This last event was regarded as confirming the diagnosis arrived at, even though no great increase in the urine passerl took place, owing, it was supposed, to the gradual nature of the subsidence.

The patient was soon after admitted into the Incurable Hospital, and I thought I had seen the last of her, but not so, for early in May of this year she returned to me saying she was worse than ever, and referring all her troubles to the abdomen. The tumour that I have already described was again present, but rather lower down than before, reaching now almost to Poupart's ligament, and with a little difficulty the hand could be pressed backwards between its upper border and the ribs. Both upper and lower borders felt rounded to the touch. It was fairly movable in a vertical direction, but not at all horizontally, and once more was dull at the side and the back, but resonant in front. The differential diagnosis between renal, splenic, and other tumours was discussed at length, and the original diagnosis of hydro- or pyonephrosis was adhered to, but with the strong suspicion now that a pyonephrosis was present. The reasons for suspecting pus were as follows :-(1) The patient's temperature, which was above normal on admission, became intermittent and "septic" in type soon after, ranging often from $97^{\circ} \mathrm{F}$. to $103^{\circ} \mathrm{F}$. within twenty-four hours. (2) The patient began to suffer from daily rigors, many of them of a severe type. (3) There was now a considerable amount of pus in the urine (catheter specimens), and no tubercle bacilli were present. The urine was acid in reaction. (4) There 
was much sweating at night, and the whole aspect of the patient was indicative of combined pain and toxrmia. The only point, in fact, against an accumulation of pus was the constant absence of any leucocytosis, or even relative increase in number of neutrophile leucocytes. It was not, however, felt that this last was a sufficient contra-indication to operation, more especially as the patient herself was clamorous for something to be done to relieve her, and accordingly it was decided to explore the region from the back, and to drain the supposed crst.

Mr. Stoney operated on Mar 16, and on cutting down found the kidney apparently normal, while the tumour lay in front of it, and turned out to be a displaced, movable and enlarged spleen, axially rotated in the manner already referred to. The patient was not sufficiently strong to warrant any attempt to deal with the spleen, and accordingly the wound was closed. A good recovery was made, but, unfortunately, without improvement in the pain complained of, and the patient ultimately left the City of Dublin Hospital to return to the Incurables. Before leaving the tumour was found to be displaced forwards a little, doubtless as a result of the operative manipulations.

In thinking over the case the most difficult symptom that I had to account for was the extraordinary variation in size which took place in the tumour, and actually under my own observation, and it was not till I discovered Mr. Bland Sutton's paper that I was able to understand it. In this paper the author states that he has seen an engorged spleen shrink to one-third its bulk during the course of a laparotomy, and also refers to a spleen which during life extended from the floor of the pelvis to midway between the umbilicus and ensiform cartilage, and which, on being excised, contracted in fifteen minutes to a length of six inches, and in six hours to about normal size. Doubtless a twisting and untwisting of the pedicle could allow similar changes to occur, and, moreover, when the organ got smaller it would more easily return to its normal place. The resemblance thus given to an intermittent hydronephosis is obvious, and, in consequence, the 
symptom deserves more attention than has hitherto been paid to it.

The origin of the pus and the cause of the intermittent temperature and of the rigors were not cleared up by the operation, and the only explanation that I can offer is that a pyelitis was present, without distension of the pelvis of the kirney, and was responsible for both pus and toxæmic phenomena. The lessened flow from the left kidney, as compared with the right, was, I presume, due to pressure upon the ureter by the enlarged and displaced spleen.

Looking over reported cases of wandering spleen that resembled hydronephrosis I can find only one or two in which marked variation in size is referred to, and perhaps the one most closely resembling the case described above is that reported by Dr. Lucy in the British Medica! Journal for July 14,1906 . Cases of this nature are, however, unfrequent, as compared with those in which a pelvic tumour is simulated, for during the last ten years not more than four or five reports in all are to be found.

As regards the rtiology in this case, I think the continued influence of the movable kidney and of cardiac congestion must be held responsible. That it was the kidney which was to be felt slipping up and down when the patient first came under notice I have not the least doubt, and the way in which this would lead to mobility of the spleen is easily conceivable from an anatomical point of view. The pain, which is often present in these cases, is presumably the result of distension of the peritoneal covering of the viscus, and of dragging upon the various ligaments.

Into the question of treatment for movable spleen I need not enter further than to say that opinion seems pretty evenly divided between splenectomy and splenopexy. The former has most frequently been practised, and has the advantage 
of yielding a permanent cure. In the case of the latter method recurrence has been more than once recorded. As already stated neither treatment was justifiable in the present instance.

\section{REFERENCES.}

1 Freuden berg. Berlin Klin. Woch. 1896. P. 659.

${ }^{2}$ Grieffenhagen. Cent. für Chirurg. 1897.

${ }^{3}$ Ssawiljew. Deutsch. Med. Zeitung. 1901. No. 65.

${ }^{4} \mathrm{H}$. Gray Croly. British Medical Journal. 1896.

${ }^{5}$ Bland Sutton. British Medical Journal January 16, 1897. 